\title{
Upgrading In-Circuit Test of high density PCBAs using electromagnetic measurement and Principal Component Analysis
}

\author{
Nabil El Belghiti Alaoui ${ }^{\text {a,b,** }}$ \\ Alexandre Boyer ${ }^{\mathrm{b}}$ \\ Patrick Tounsi ${ }^{\mathrm{b}}$ \\ Arnaud Viard ${ }^{\mathrm{a}}$
}

${ }^{\mathrm{a}}$ ACTIA Automotive, 5 Rue Jorge Semprun, 31400, France

${ }^{\mathrm{b}}$ Laboratoire CNRS, LAAS, 7 avenue du colonel Roche, Univ. de Toulouse, INSA, F-31400

Keywords - PCB assembly production test, magnetic sensors, In-circuit test, contactless testing, Principal Component Analysis, outlier detection 
Abstract - With the density increase of today's printed circuit board assemblies (PCBA), electronic test methods such as in-circuit test (ICT) reached their limits. In the same time the requirements of high reliability and robustness are greater. Original equipment manufacturers are obliged to reduce the number of physical test points and to find betteradapted test methods to keep adequate test coverage. Current test methods must be rethought to include a large panel of physical phenomena that can be used to detect- electrical defects, absence, wrong value of components, absence and shorts without using test points on the board under test (BUT).

In this paper, a test set-up based on the measurement of electromagnetic signature to diagnose faulty components contactlessly is presented. The technique consists in using magnetic field probes, which detect the field distribution over powered sensitive components. To evaluate the relevance of the method, reference EM signatures are extracted from fault-free circuits, which are compared to those extracted from a sample PCBA in which we introduced a component level defect by removing or changing the value of critical components. For more robust detection of multiple defect scenarios, the principal component analysis (PCA) method is used as an outlier detection algorithm.

\section{Introduction}

During the assembly process of printed circuit boards (PCB), defects such as wrong value components, missing components, unwanted open circuits or short circuits may appear. Manufacturers continually look for faster, more accurate and more economical ways to identify this kind of defects. That's why performing automated testing of dense populated PCBs is a mandatory and cost effective solution to ensure manufacturing quality control.

Testing today's populated PCBs is becoming increasingly challenging and more expensive as the use of small size surface mounted devices (SMD) is becoming predominant. The emergence of new technologies as High-Density Interconnect (HDI), embedded chips and Sequential Build-Up (SBU) circuit boards will even further increase the challenge for the test business.

Conventional techniques for automated PCBA testing involve applying signals through a number of test pins and measuring the output signals on the other test pins. Functional testing can be performed by energizing the PCBA, applying a predetermined number of input signals, and determining whether the proper output signals are generated by the circuitry on the BUT [1]. Alternatively, for a high volume manufacturing (HVM) process, a PCBA is tested primarily on a "bed-of-nails" fixture called incircuit tester (ICT). It comprises pins called "nails" which directly contact the metallic traces on the BUT so that selected input signals may be applied at various test points (TP) on the PCB, and corresponding output signals can be measured on other TPs. This requires several physical TPs on the PCB traces which can compromise the integrity of the tested signals.

This widely used classical technique requires tight mechanical tolerances for the board layout, easily accessible test points and restricts the frequency band at which a board can be tested [2], which cannot be afforded anymore on a state of the art PCBA. Starting from this need, the idea of taking advantage of the HVM nature of the ICT and trying to upgrade it with contactless probes to meet current test challenges have come. In this paper, we present a new testing approach using electromagnetic (EM) near field sensors (NFS) to test populated PCBs. A state of the art comparison presented in our previous article [3] shows that there's no method existing today that takes advantage of the classical ICT characteristics and upgrades its "probing by contact" structure to a contactless bed-of-nails structure to test assembled PCBs.

The approach presented here aims to upgrade in-circuit testers by suppressing some of the ICT testing nails which are contact probes used to perform electrical test of the components mounted on the board, and replace them with contactless probes. The principle of the proposed method is explained in section 2 in order to understand its large scale application. To this aim and to prove the effectiveness of the method, we chose a DC/DC buck converter module as a case study. The converter module, the experimental test bench and the used NFS are described in section 3. Simulations of value defect scenarios have been carried out on Cadence Orcad and validated by measurements on DC/DC converter boards in which we introduced controlled value defects on the input decoupling ceramic capacitors, output capacitors and output filter inductance. Simulation and experimental results are reported in section 4 to prove that component value defects can lead to measurable variations of EM signatures.

As explained in section 5, for more robust defect detection on component level and thereafter PCBA level, we need to take into account correlations between the measured EM signatures and the different sources of uncertainty that affect the signatures measurements. An outlier detection algorithm based on Principal Component Analysis (PCA) is proposed and detailed in section 6. This defect detection method is carried out on MonteCarlo simulation data of the DC/DC buck converter according to the simulation set-up described in section 7. Results are reported in section 8. Finally, discussion and perspectives about the upgrading of the ICT and the detection method are provided in conclusion.

\section{Description of the proposed testing approach}

To improve the effectiveness and accessibility of current ICTs and gain accessibility to components that could not be tested without placing numerous 
test points, we propose in this article a new test approach based on EM inspection using near field sensors as an upgrade to the classical In-circuit testers. With magnetic field measurements above PCBA components, contactless information about current distribution can be obtained without access limitation on the PCBA surface.

\subsection{Principle}

The principle of this method (see Fig.1) is to measure the EM near field distribution directly over the center of a powered component contactlessly and compare the measured electromagnetic signature (EMS) to a database of correct signatures preestablished on fault-free PCBAs. The measured electric and magnetic fields (EMF) are generated by the distribution of charges and currents respectively in the components mounted on the BUT. Accurate and repeatable measurements of these fields produce a specific time and frequency domain signature for each critical component, which can be extended to a complete operational block, and then to the complete board in a further application. Such signatures are then compared to a pre-established non-faulty signature pattern of the same type of board to determine whether the BUT is faulty or not and pinpoint exactly where the faulty component is located knowing the position of the NFS on the board.

Thus firstly, the board or the operational block of the board is powered and operates normally. The EM near-field distribution generated by every "critical" component is then detected using NFS mounted directly over these components in a bed-ofnails structure. The registered signature specific to the component and excitation conditions of the electronic block is registered. Subsequently, the measured signature is compared with sample signatures of the same block measured on multiple non-faulty boards, which were registered in the same excitation conditions to determine whether the response is in conformance with the known reference.

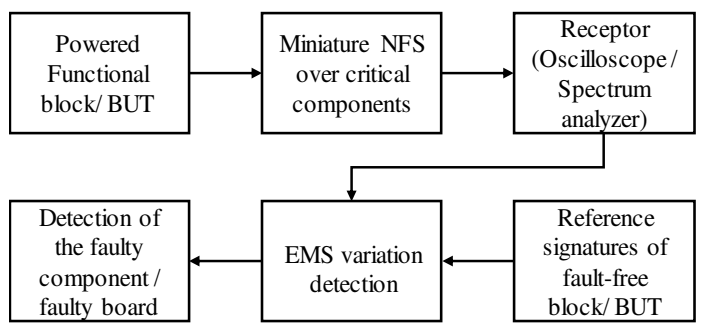

Fig. 1. Principle of the near field probing test approach

\subsection{Types of detected assembly defects}

The defects that can be detected with this method are at component level. In other words, it is supposed that the bare PCB is fault free guaranteed from the manufacturer, or had already been tested and certified non-faulty. Assembly defects over critical components that can be detected:

- presence of components,
- polarity for components that exhibits current change when mounted in reverse polarizations,

- value of components,

- a wrong package that can change the height of the component,

- overstressed/overheating components which could be degraded

- solder defects (open and shorts).

These components must be carefully chosen in advance in order to establish design for testability rules (DFT) to minimize the probe count and maximize fault detection on a functional block level.

\section{Description of the case study: DC/DC buck converter}

To validate our approach, we chose a DC/DC buck converter because of the important transient currents crossing the components when the module is powered. Components such as input/output filtering capacitors, MOSFET and inductors radiate a significant high frequency magnetic field in the near field region due to the large transient currents crossing them. The currents and the induced magnetic fields are related to component values, package and mounting. From the analysis of measured magnetic fields, the presence and the location of assembly defects or wrong components can be detected.

This case study is a typical test scenario of a DC/DC converter module in a power management block of an industrial high density PCBA.

We chose an off-the shelf evaluation board of a synchronous DC/DC buck converter module with a fully integrated controller [4] to run tests, a basic electrical diagram is given in figure 2 . The powering conditions for test are as mentioned in Table 1.

Table 1

Powering conditions for test

\begin{tabular}{ll}
\hline Input voltage $\mathrm{V}_{\text {in }}$ & $20 \mathrm{~V}$ \\
Output voltage $\mathrm{V}_{\text {out }}$ & $12 \mathrm{~V}$ \\
Load current $\mathrm{I}_{\text {Load }}$ & $3 \mathrm{~A}$ \\
Frequency & $250 \mathrm{kHz}$
\end{tabular}

\subsection{Simulated defect scenarios}

The defect scenario used to validate this approach is the detection of a wrong value of an input decoupling capacitor, output capacitor and filtering inductor.

The approach is tested initially in simulation, and then in measurement in order to prove the relevance of the measured EM signature variations.

We modeled the DC/DC buck converter evaluation board on Allegro Design Entry [5] using the PSPICE model of the controller given by the manufacturer (see Fig.2). Estimated values of parasitic elements of each critical component were used to give a more realistic simulation result. Then, we ran multiple parametric simulations with four different values of each input capacitor (see Table 2). The simulation aims at evaluating how the derivative 
of the current in each input capacitor reacting to a change of value of an input capacitor. The derivative of the current flowing through a component is related to the magnetic field measured with a commercial NF probe over this specific component, as described in 3.3 .

\section{Table 2.}

Input capacitor values in simulation and experiments

\begin{tabular}{lll}
\hline $\begin{array}{l}\text { Input } \\
\text { capacitors }\end{array}$ & $\begin{array}{l}\text { Correct } \\
\text { value }(\mu \mathrm{F})\end{array}$ & $\begin{array}{l}\text { Incorrect } \\
\text { values }(\mu \mathrm{F})\end{array}$ \\
\hline C8 & $2.2 \mu \mathrm{F}$ & $1,1.5,3.3,15$ \\
C9 & $2.2 \mu \mathrm{F}$ & $1,1.5,3.3,15$ \\
C10 & $2.2 \mu \mathrm{F}$ & $1,1.5,3.3,15$ \\
C11 & $2.2 \mu \mathrm{F}$ & $1,1.5,3.3,15$ \\
\hline
\end{tabular}

\subsection{Test procedure: Experimentation}

We reproduced the same scenario described earlier with the same values on the evaluation DC/DC buck converter module. We changed the value of each input and output filtering capacitor on the board several times by soldering and removing a different value capacitor separately. Then, we collected the time domain signatures over each capacitor using an oscilloscope for each of the four values evaluated. Collected signatures of each capacitor were analyzed to evaluate their variance compared to the reference signatures.

\subsection{Near-field sensors description \\ 3.3.1. Near field probes}

Measuring the time domain $\mathrm{H}$-field signatures over the powered (see Table 1) DC/DC buckconverter was carried out using a commercial mini (resolution $<1 \mathrm{~mm}$ ) Near-Field probe [6] (see Fig.4) which measures the derivative of the lateral magnetic fields over the components in the range from 30 $\mathrm{MHz}$ to $3 \mathrm{GHz}$. The probe was directly connected to a digital oscilloscope with $50 \Omega$ input impedance. The NFP was then freely and accurately moved over every input capacitor in near-field region at a $2 \mathrm{~mm}$ distance of the center of the component using an automatic computer controlled scan table with a distance precision of $25 \mu \mathrm{m}$ (see Fig. 3).

\subsubsection{GMR Sensors}

Near field probes have a high sensitivity when measuring high frequency fields, but, low frequency fields are not detected accurately. To compensate the lack of sensitivity in low frequency measurements, another type of magnetic-field sensors is used: giant magnetoresistance (GMR) sensor.

The GMR effect discovered in 1988 is related to field dependent changes in resistance that can be observed in thin-film ferromagnetic/non-magnetic metallic multilayers. The term GMR was coined due to the large change of resistance (10 to $20 \%$ ) of the thin-film materials. GMR sensors have taken an important role due to their small size, high signal level, high sensitivity, large frequency response and low cost [7].

Contrary to the NFP, this sensor provides better sensitivity for relatively low frequency magnetic fields (up to $1 \mathrm{MHz}$ ) [7]. In a DC/DC converter, such low frequency magnetic field dominates above the output inductor. The low frequency current ripple crossing the inductor can be measured precisely with a GMR sensor to detect any variation of the inductance.

In this study, a commercial multilayer GMR sensor with a sensitivity of $5.4 \mathrm{mV} / \mathrm{V} / \mathrm{A}$ for frequencies up to $100 \mathrm{KHz}$ from NVE spintronic is used [8]. Its use can be extended up to $400 \mathrm{kHz}$ because its sensitivity is only $3 \mathrm{~dB}$ attenuated.

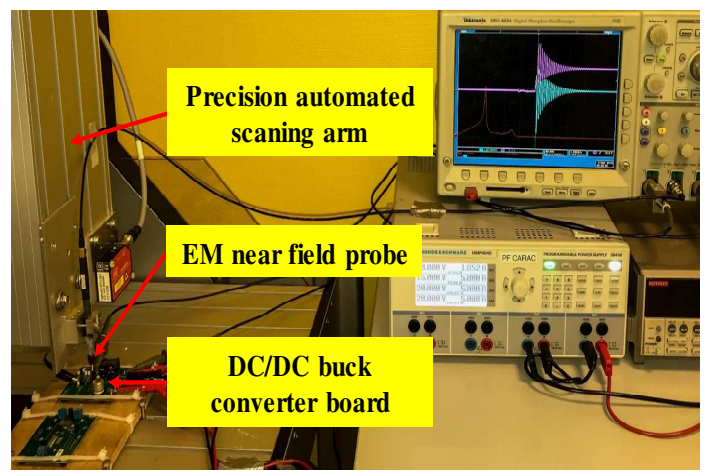

Fig. 3. Test bench set-up

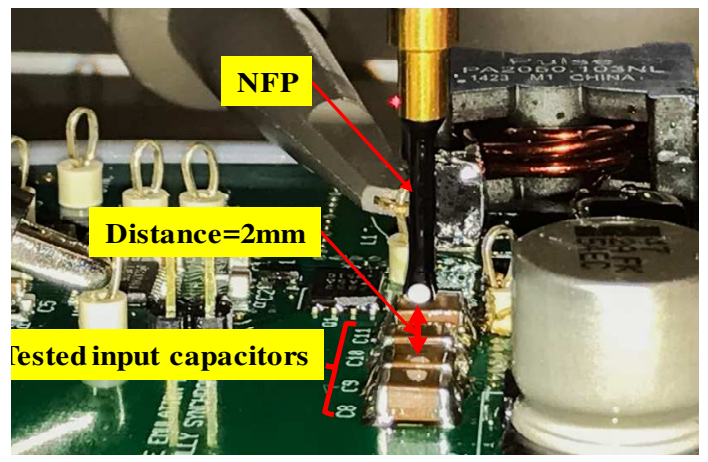

Fig. 4. Magnetic NFP and the probed components 


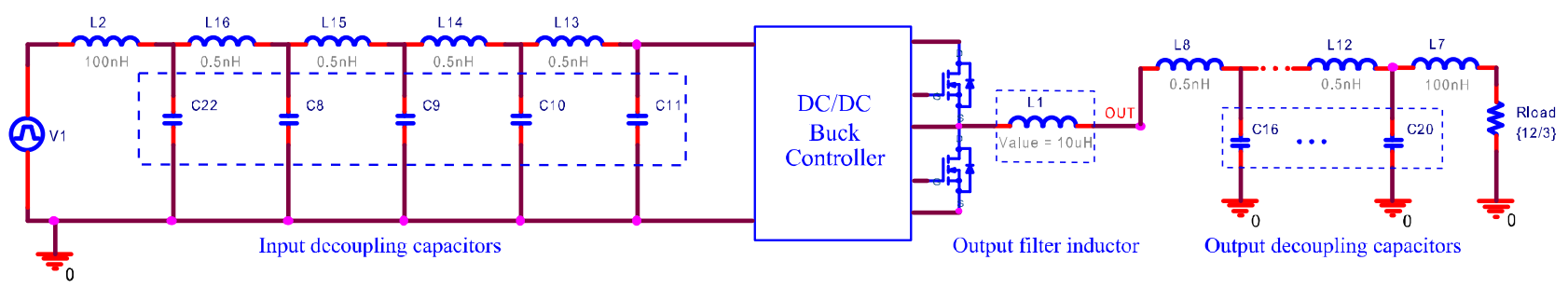

Fig. 2. Simplified schematic of simulation

\section{- Test conditions}

We used the commercial GMR sensor to test a 2525 SMD inductor mounted on a different DC/DC Buck converter evaluation module [9] (see Table 3).

The measurements were taken at a distance of $2 \mathrm{~mm}$ from the surface of the inductor with four different values (see Fig. 5). The converter was powered on, and the output load current was constant at $3 \mathrm{~A}$.

Table 3

Output inductance test conditions

\begin{tabular}{ll}
\hline $\mathrm{V}_{\text {in_DC/DC }}$ & $10 \mathrm{~V}$ \\
$\mathrm{~V}_{\text {out_DC/DC }}$ & $1.2 \mathrm{~V}$ \\
$\mathrm{~V}_{\text {supply_GMR }}$ & $20 \mathrm{~V}$ \\
$\mathrm{~F}_{\text {sw }}$ & $400 \mathrm{KHz}$ \\
$\mathrm{I}_{\text {Load }}$ & $3 \mathrm{~A}$ \\
Inductor reference value & $\mathrm{L}=0,47 \mu \mathrm{H}$ \\
\hline Inductor wrong values & $\mathrm{L}=0,22 \mu \mathrm{H}$ \\
to be detected & $\mathrm{L}=82 \mu \mathrm{H}$ \\
& $\mathrm{L}=1,5 \mu \mathrm{H}$ \\
\hline
\end{tabular}

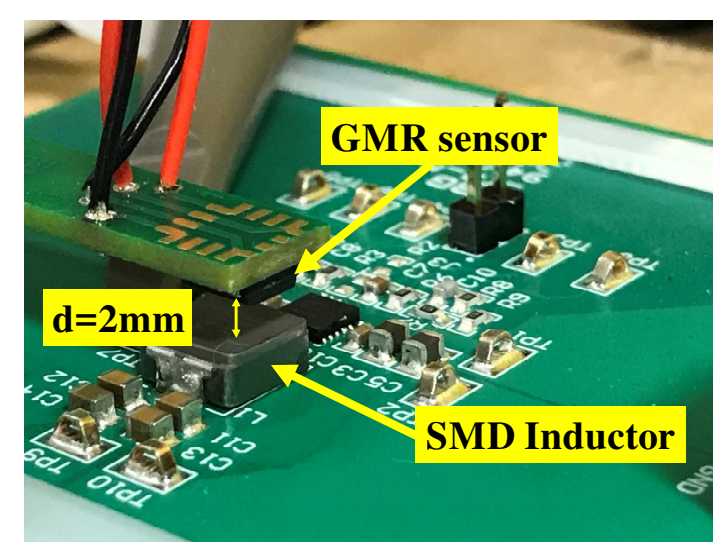

Fig. 5. Image of the GMR sensor and the measured SMD inductor

\section{Simulation and experimental results \\ 4.1. Test of the input decoupling capacitors 4.1.1 Reference signatures}

The reference signature is the magnetic field captured by the NFP over each input capacitor when it has a correct value.

Figure 6 shows that only around a specific frequency we obtain the same significant signature in simulation and measurement over input capacitors C8, C9, C10, and C11.

The noticeable difference in amplitude and frequency scales between simulation (dashed lines) and measurement (solid lines) is due to assumptions made on the parasitic parameters of the components in the simulation model to be more considered in a future work. This is also due to the coupling parameter of the NFP that wasn't taken into account in the simulation model.

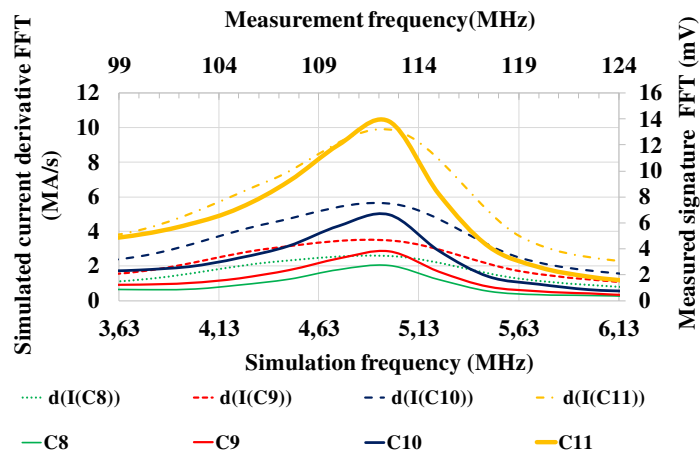

Fig. 6. Simulated current derivatives FFT of input capacitors (dashed lines), (vs) measured EM signatures FFT on the buck converter PCB

\subsubsection{Signatures with wrong values of the input capacitors}

In concerns of conciseness, only the signatures measured over all input capacitors induced from the variation of the value of the input capacitor $\mathrm{C} 8$ will be presented in this section (see Fig. 7 and Fig. 8).

The dashed lines show the small signature variations of the unchanged input capacitors (C9, $\mathrm{C} 10, \mathrm{C} 11)$. Bold lines are the variation of $\mathrm{C} 8$ signatures when its value changes. Results from simulation and measurements show that the magnetic signature of C8 varies significantly, while other capacitors signatures change more slightly.

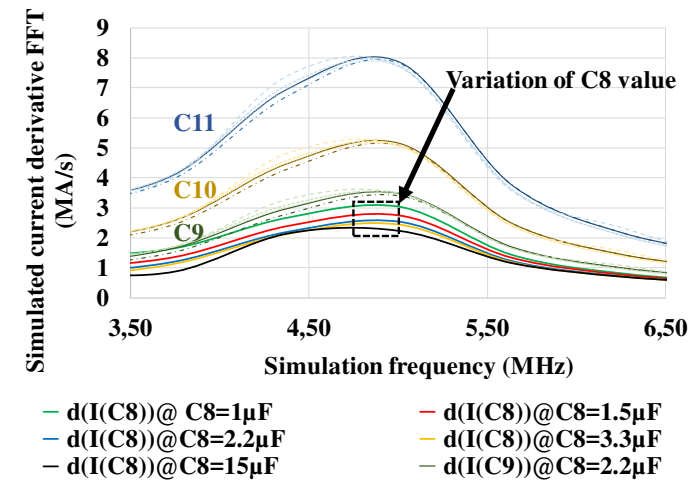

Fig. 7. Simulated current derivatives FFT of input capacitors when $\mathrm{C} 8$ varies 


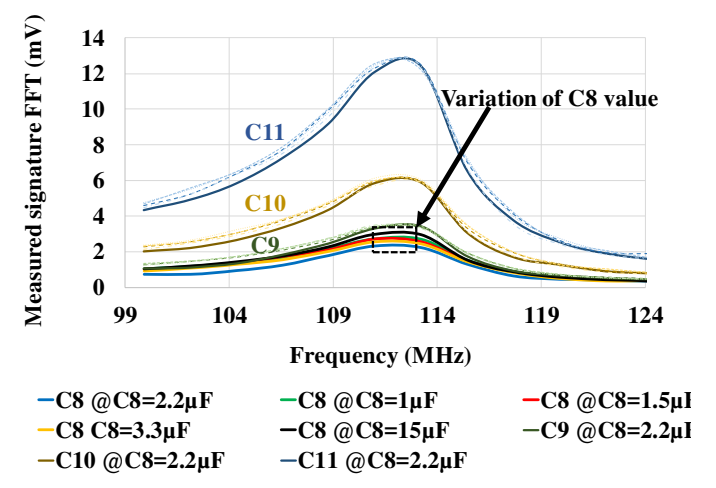

Fig. 8. Measured magnetic signatures FFT of the input capacitors when $\mathrm{C} 8$ varies

\subsubsection{Comparison and analysis}

From the measurement results we can see that the capacitor value change induces a significant variation on the amplitude spectrum at around $110 \mathrm{MHz}$ of the EM signature of the capacitor being changed. This frequency is due to the resonance of the input capacitors with the parasitic elements of the switching stage of the converter, which depend on the board under test and the mounted devices.

This is still true for all input capacitors when we change their value. They all exhibit a significant variance of their signature FFT amplitude at around 110MHz. We summarized all simulation and measurement results of all changing capacitors $(\mathrm{C} 8, \mathrm{C} 9, \mathrm{C} 10, \mathrm{C} 11)$ in the graphs below (see Fig. 9.a and 9.b).

A first simple mathematical tool we used to prove detection of wrong mounted components values through their magnetic signatures is the root mean squared deviation (RMSD) (see equation (1)). This statistical measure is used to quantify the amount of dispersion of the magnetic signatures measured over each capacitor around a reference signature at the resonance frequency. A low RMSD indicates that the signatures tend to be close to the reference signature, while a high RMSD indicates that they are spread out over a wide range of values. The graphs shows that the most scattered signatures around the reference are those of the decoupling capacitor for which the value was changed.

$$
\begin{array}{ll}
R M S D_{\text {ref }}=\sqrt{\frac{1}{N-1} \sum_{i=1}^{N}\left(X_{i}\left(f_{\text {res }}\right)-X_{\text {ref }}\left(f_{\text {res }}\right)\right)^{2}} \\
N: \quad \begin{array}{l}
\text { number of magnetic signatures for } \\
\text { each capacitor value } \\
\text { magnetic signature over a wrong } \\
\text { value capacitor at the resonance } \\
\text { frequency } \\
\text { magnetic signature corresponding to } \\
\text { the reference value of the capacitor at } \\
\text { the resonance frequency }
\end{array} \\
X_{\text {ref }}\left(f_{\text {res }}\right): &
\end{array}
$$

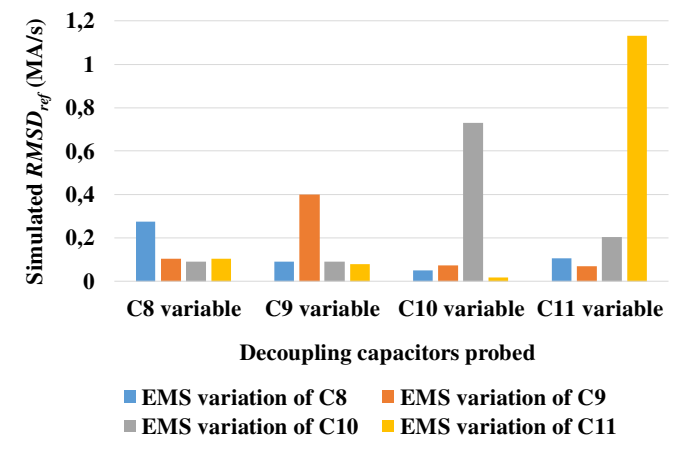

Fig. 9. a. RMSD of the simulated FFT amplitude of the magnetic signatures in each case where the value of one input capacitor is changed separately

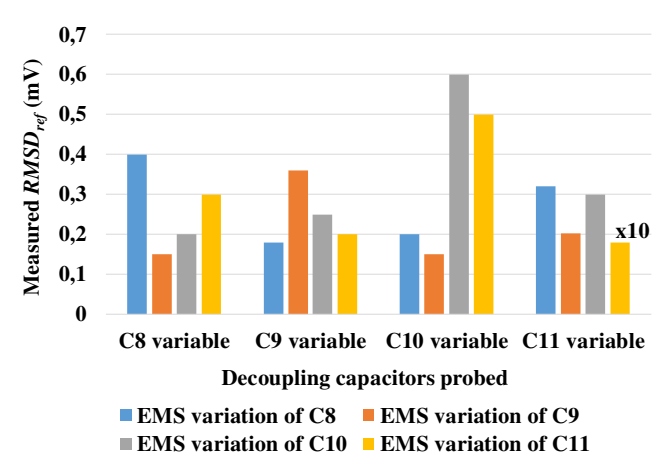

Fig. 9. b. RMSD of the measured FFT amplitude of the magnetic signatures in each case where the value of one input capacitor is changed separately

\begin{abstract}
${ }^{\mathrm{x} 10}$ The value of this deviation is $1,8 \mathrm{mV}$, it was divided by 10 to fit in the comparison graph.
\end{abstract}

We can see that there is a coherence between simulation and experimental results. The RMSD of the value-changed capacitor is always the highest, which allows the detection of a wrong value capacitor through the comparison with a reference signature without the need of using test points. A robust detection method will be presented in section 6 .

\subsection{Test of the output filter capacitors}

The output capacitance of a switching DC/DC converter is a vital part of the overall feedback system. The energy storage inductor and the output capacitors form a second-order low-pass filter. The output filter's inductor therefore limits the current slew rate. When the amount of current required by the load changes, the initial current deficit must be supplied by the output capacitors until the regulator can meet the load demand [10].

In order to measure magnetic field signatures over these output capacitors we need to emphasize on their effect by pushing them to provide a high transient current to the load.

To do so, we designed a load that provides a current step by switching the output current of the converter from $50 \mathrm{~mA}$ to $2.5 \mathrm{~A}$, with a rising time of $1 \mu \mathrm{s}$ and a falling time of $0.5 \mu \mathrm{s}$ [3].

4.2.1 Signatures with wrong values of the output capacitors 
Magnetic field signature measurements with NFP over each changing output capacitor (see Table 4) show clearly which capacitor's value is being changed. Only the signatures measured over the accessible top board capacitors (C16 and C20) are presented here (see Fig. 10 and Fig. 11).

The dashed lines show the small signature variations of the unchanged output capacitors. Bold lines are signature variations of the changed output capacitors, in this case the capacitor $\mathrm{C} 16$ and $\mathrm{C} 20$.

Table 4

Values used for the output capacitors

\begin{tabular}{lll}
\hline Input & Correct & Incorrect \\
capacitors & value $(\mu \mathrm{F})$ & values $(\mu \mathrm{F})$ \\
C16 & $47 \mu \mathrm{F}$ & $22,33,68$ \\
C20 & $22 \mu \mathrm{F}$ & $10,15,33$ \\
\hline
\end{tabular}

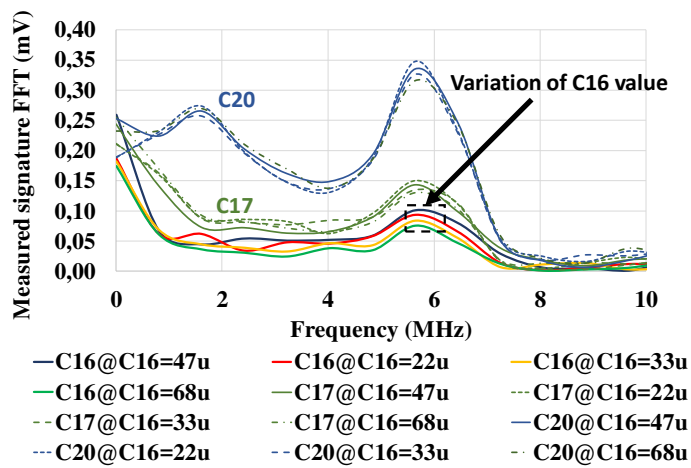

Fig. 10. Measured magnetic signatures FFT of the output capacitors when $\mathrm{C} 16$ varies

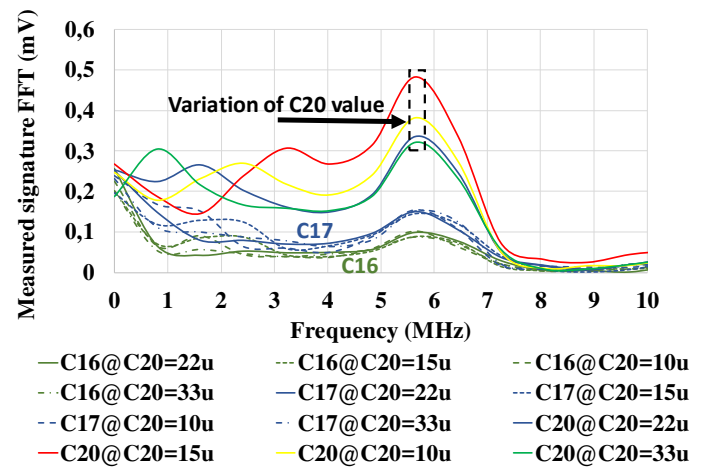

Fig. 11. Measured EM signatures FFT of the output capacitors when $\mathrm{C} 20$ varies

We can clearly distinguish the capacitor with a wrong value from the amplitude of its signature deviating from the reference one. This measured signature is the resonance of the loop composed of the output capacitors and the current step PCB parasitic elements (trace inductances and output load parasitic capacitances). In this particular case, we observed the resonance at $5.5 \mathrm{MHz}$.

\subsection{Test of the output filter inductance}

The results presented in figure 12 show the possibility to detect variations of the value of the inductor using a GMR sensor. The peak-to-peak amplitude of the sensor's output voltage doubles as the value of the inductor is divided by 2 , which is coherent since the sensors output has a linear relationship with the $\mathrm{AC}$ magnetic field $(B)$ which is proportional to the current ripple $\left(\Delta I_{L}\right)$ in the inductor (see equation (2)).

$$
\begin{aligned}
& V_{\text {ou }_{G M R}}=S_{\text {dynamic }}(f) \times B\left(\Delta I_{L}\right) \\
& \Delta I_{L}=\frac{1}{F_{s w} X L} V_{\text {out }_{D C ~} / D C}\left(1-\frac{V_{\text {out }} D C / D C}{V_{\text {in }}}\right)
\end{aligned}
$$

$F_{s w}$ is the converter's switching frequency and $L$ is the value of the filtering inductance. All measurements are in raw conditions. No shielding, filtering or amplification were used.

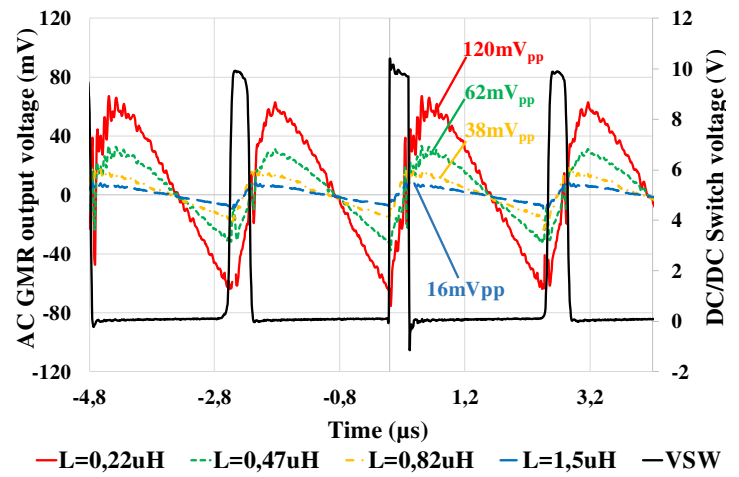

Fig. 12. Output voltage of the GMR sensor showing the variation of the inductor's value

The sensor's AC output voltages reflects the waveform of the current ripple in the inductor, which is inversely related to the value of the inductance (see equation (3)). Table 5 resumes the results presented in Fig. 12.

The measurement of the magnetic field above the inductor, and the comparison with the measurement on a reference sample offer a simple method to detect a bad mounted inductor value without the need for test points.

Table 5

Sensor output voltage according to inductor's values

\begin{tabular}{ll}
\hline \multicolumn{1}{c}{$\begin{array}{c}\text { Value of the inductance } \\
(\mu \mathrm{H})\end{array}$} & \multicolumn{1}{c}{$\begin{array}{c}\text { Sensors output voltage } \\
\left(\mathrm{mV}_{\mathrm{pp}}\right)\end{array}$} \\
\hline 0,22 & 120 \\
0,47 (reference value) & 62 \\
0,82 & 38 \\
1,5 & 16 \\
\hline
\end{tabular}

\section{Discussion}

In section 4.1.3 a simple statistical indicator was used to prove the concept of our test approach. It showed that the dispersion of wrong capacitor EM signatures around the reference signature in a simple defect scenario in which we introduced one wrong value component at a time is higher in comparison with other capacitors with unchanged values.

In contrast with the PCB assembly industry, in order to test assembled PCBs in production using ICT with our approach, we must prove our solution in a scenario that takes into account multiple defects in the same capacitor set and integrates different sources of uncertainty, which affects the magnetic signature measurement (see Fig. 13). 


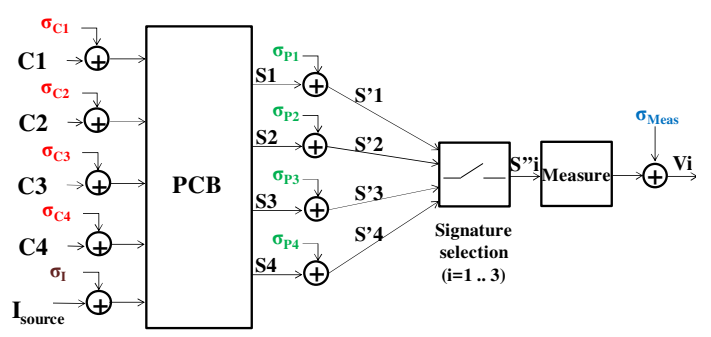

Fig. 13. Principal sources of uncertainty that affect magnetic signature (S to S4) measurements with NF probes for each tested capacitor ( $\mathrm{C} 1$ to $\mathrm{C} 4)$

The different sources of uncertainty which can affect measurement results are:

- Dispersion of component values within tolerance range: $\sigma_{(1,2,3,4)}$

- NFS position over the components $\mathrm{C} 1$ to $\mathrm{C} 4$ and solder process variations: $\sigma_{(\mathrm{P} 1, \mathrm{P} 2, \mathrm{P} 3, \mathrm{P} 4)}$

- Current $I_{\text {source }}$ variations due to surrounding magnetic field coupling: $\sigma_{\mathrm{I}}$

- Measurement error introduced by the measurement equipment: $\sigma_{\text {Meas }}$

All these error sources must be taken into account when processing the measured signatures.

The main uncontrolled uncertainties that vary randomly from $\mathrm{PCB}$ to another are the component value dispersion within the tolerance range and excitation current variations. It is clear that adding these uncertainties in our system and trying to detect defects with a simple dispersion indicator as done in the first scenario, cannot give a result with a high degree of confidence. Furthermore, signatures measured over each component are partially correlated. These correlations follow complex laws difficult to predict, which makes a defect indicator based solely on RMSD insufficient to account for these correlations.

Another reason to use a different fault detection method is the large number of measured signatures on an industrial assembled PCB during ICT. This will make the process of detecting faulty components using RMSD very complex and time consuming. Hence, the necessity to use a more robust detection algorithm. In the next part, a defect detection method is presented.

\section{Defect detection algorithm}

The definition of a component defect in our case is every signature measurement which deviates significantly from the reference. Small signature deviations which result from capacitor value tolerance and current variations must not influence the detection. In other words, the defect detection algorithm must be able to recognize these variations in a signature measurement and distinguish them from faulty signatures. Only measurements which deviates significantly from tolerance ranges should be taken as defect signatures.

Measuring signatures over $N$ components mounted on a PCB forms an $N$ dimensional space in which abnormal signature values will be single data points that lay far from the rest of the distribution, and therefore considered as outliers [11].

Figure 14 describes the integration of outlier detection algorithm for one assembled PCB in the production test process.

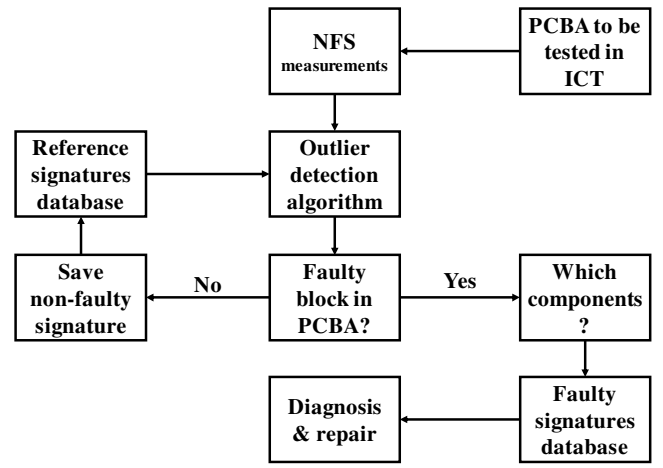

Fig. 14. Outlier detection algorithm in the production process

\subsection{Outlier detection}

In the process of collecting, processing and analyzing data in an industrial environment, outliers can come from many sources and hide in many dimensions. That is the reason why detecting defect outliers properly is of major importance to assure the quality of products.

There are several methods for detection of outliers available in the literature: parametric methods such as extreme value analysis, probabilistic and statistical modeling:

- Although they are useful when the distribution of measured values in the featured space is well known, however, they don't take correlation between variable into account which makes them useless in our case;

- non-parametric methods such as density based scan (DBscan): a really powerful clustering method when dealing with a large density of data, nevertheless weak when clusters have large differences in densities;

- linear regression models which include projection methods that model the data into lower dimensions using linear correlations. These projection methods are relatively simple to apply and quickly highlight extraneous values [12].

Principal Component Analysis (PCA) for example is one of the projection methods that take the correlation between variable into account to lower the dimensions of the featured space. It also makes it possible to analyze the influence of variation of each variable, which is very interesting in our application in order to determine which components are the source of the outliers [13].

6.2 Outlier detection using Principal Component Analysis

Analogically with the statistics domain, our measurement system has:

- One independent variable (IV), which is the dummy defect we introduce and control, in 
other words: the value of the components we vary.

- Multiple dependent variables (DV), which are the measured magnetic signatures. As we explained earlier, we can have multiple defects in the same capacitor set, which means multiple signatures that change differently at the same time. Thereby, our statistical system will have multiple dependent variables which may present a certain degree of correlation between them.

Since we can't assume a distribution of values for our measured variables, we will suppose that the system is non-parametric.

Applying our test approach in a PCBA production line, we will be surrounded by data with a large number of variables, some of which might be correlated. This correlation between variables brings about a redundancy in the information that can be gathered by the data set. Thus, in order to reduce the computational and cost complexities, we can use PCA to transform the original variables to linear combinations of these variables called principal components (PC), which are uncorrelated. In this new coordinate system, the covariance matrix is diagonal and the first PC points into the direction with the largest variance within the data set. The PCs are the eigenvectors of the covariance matrix and its eigenvalues are the variance obtained with these new variables. Given the percentage of variation that we want to be captured in the abridged data set, we can select the number of PCs to be considered without a significant loss of information.

PCA is not only a technique to reduce the number of variables but can further be used for classification purposes based on the Principal Component Scores. Using PCA in our case will allow us to classify data into 2 categories: faulty PCBs and good PCBs, which will be presented next in an application on simulation data extracted from our DC/DC buck converter model.

\section{Simulation set-up}

Using the simulation model of our DC/DC buck converter, six non-faulty PCBs are generated with differences in terms of components values. They vary randomly according to their tolerance range using a uniform Monte-Carlo simulation. Among them, a faulty PCB with one or multiple value defects in the decoupling capacitors block is inserted. We added as well a small random variation in the input supply voltage, noted V1 in figure 2.

The Principal Component Analysis algorithm was applied on simulation data using the Statistics and Machine Learning Toolbox of Matlab [14]. These data are the Fast Fourier Transform (FFT) of the currents flowing through in each input decoupling capacitor.

The PCA of raw data function implemented in Matlab does the following:

- Centers variables by subtracting their mean value.
- Does not scale data, which is not really relevant in our case because all measurements are in the same scale.

- Creates the Principal Component (PC) vector space called 'Loadings' which are equal to:

Loadings $=$ Eigenvectors $\times \sqrt{\text { Eigenvalues }}$

The eigenvectors and eigenvalues are calculated from the covariance matrix of the measurements.

- Projecting the original measurement data on the PC vector space by multiplying the measurements matrix, called data matrix, by the PC vectors. As a result, we obtain a matrix called PC scores that represents the new coordinates of each data point in the new PC coordinate system.

- The eigenvalues calculated from the covariance matrix of the data represent the variance captured by each Principal Component.

Knowing how to represent this output information graphically and interpreting them is key to determine which PCB is faulty and even more which component is responsible of the defect.

In our case, we have a data matrix of four capacitors. Thus PCA calculates four Principal Components and arranges them in a descending order of variance. In the following results we choose the three highest variance PCs in order to plot the scores in a $3 \mathrm{D}$ graph for clarity purpose.

\section{Results}

\subsection{Detection scenarios}

To be sure that PCA can detect multiple defects on the same capacitor block, several possible defect cases in the decoupling or output capacitor blocks were tested. This defect cases are summarized in Table 6. Only results from $\mathrm{N}^{\circ} 1$ and $\mathrm{N}^{\circ} 2$ defect scenarios are presented, the other cases gave similar results.

Table 6

Simulated defect scenarios

\begin{tabular}{|c|c|c|}
\hline$N^{\circ}$ & $\begin{array}{l}\text { Defect } \\
\text { scenarios }\end{array}$ & Details \\
\hline 1 & $\begin{array}{l}\text { One } \\
\text { wrong } \\
\text { value } \\
\text { capacitor }\end{array}$ & $\begin{array}{l}a \text {. The wrong value is lower than reference } \\
b \text {. The wrong value is higher than reference }\end{array}$ \\
\hline 2 & $\begin{array}{l}\text { All wrong } \\
\text { value } \\
\text { capacitors }\end{array}$ & $\begin{array}{l}\boldsymbol{c} \text {. All wrong values are lower than reference } \\
\boldsymbol{d} \text {. All wrong values are higher than reference } \\
\boldsymbol{e} \text {. At least one wrong value is lower than } \\
\text { reference } \\
f \text {. At least one wrong value is higher than } \\
\text { reference }\end{array}$ \\
\hline 3 & $\begin{array}{l}\text { Two } \\
\text { wrong } \\
\text { value } \\
\text { capacitors }\end{array}$ & $\begin{array}{l}\text { g. first wrong value is higher than reference; } \\
\text { second is lower than reference } \\
\boldsymbol{h} \text {. Both wrong values are higher than reference } \\
\boldsymbol{i} \text {. Both wrong values are lower than reference }\end{array}$ \\
\hline 4 & $\begin{array}{l}\text { Three } \\
\text { wrong } \\
\text { value } \\
\text { capacitors }\end{array}$ & $\begin{array}{l}j \text {. All wrong values are higher than reference } \\
\boldsymbol{k} \text {. All wrong values are lower than reference } \\
\boldsymbol{l} \text {. At least one wrong value is higher than } \\
\text { reference } \\
\boldsymbol{m} \text {. At least one wrong value is lower than } \\
\text { reference }\end{array}$ \\
\hline
\end{tabular}




\subsection{PCA detection frequencies}

Contrarily to the detection method reported earlier in this article, the frequency on which we are going to detect signature variation, does not show up at first sight. A frequency range in which the signal to noise ratio (SNR) was at minimum for all measures was selected, and a PCA was run on the ten first frequencies of the current's FFT spectrum for each and every defect scenario. A cross reference between all the results obtained showed that the detection is always significant for the even multiples of the harmonic frequency at $250 \mathrm{kHz}$. This frequency is therefore chosen to run a PCA. In a more general application case, this frequency is to be defined upstream with the PCB designer.

\subsection{Defected PCBs detection}

A graphical presentation of the PC scores shows clearly which $\mathrm{PCBs}$ are faulty and consider them as outliers (see Fig.15).

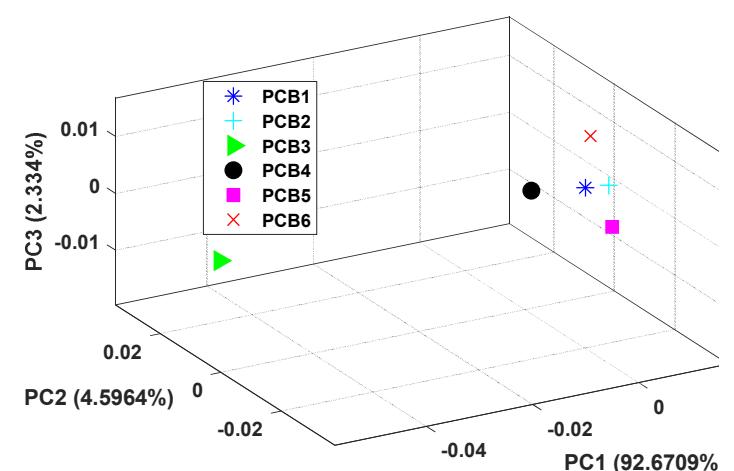

Fig. 15. PCA results for 6 PCBs with one faulty PCB: PCB3

The first Principal Component (PC1) captures the most variation of the data- $92.67 \%$ - which is the ratio between the eigenvalue associated to PC1 and the sum of all eigenvalues. This ratio describes the contribution of a particular PC to the dispersion of the data.

However, a question arises: which metric to use in order to characterize the detection of this outlier on the PCA score plot?

A characteristic quantity of an outlier point in the PCA score plot is its distance from the non-faulty PCB cluster centroid which is predefined based on PC1, PC2 and PC3 score values in a prior phase. Expressing this distance as a function of the standard deviation of the non-faulty PCB cluster can define a detection threshold of an outlier data point.

A statistical quantity which can be used in this case is the Z-score. It describes how much a measurement $M$ deviates from the mean $\mu$ of $\mathrm{N}$ sample (see equation (5))

$$
Z_{\text {score }}=\frac{M-\mu}{\sigma}
$$

It is generally involved with Chauvenet's criterion [15] for rejecting or accepting deviated measurements but only when assuming a normal distribution of the data. In our case the data distribution is not known which prevent from using Chauvenet's criterion to set a detection threshold.

To define a viable outlier detection threshold, a worst case detection scenario was adopted.

This scenario consists of choosing capacitor defective values very close to the tolerance range which is a non-defect value range and assess the Z-score behavior. The test cases are presented in Table 7 and the corresponding results in figure 16.

Table 7

Simulated worst case defect scenarios of capacitor

\begin{tabular}{ll}
\hline Wrong values $(\mu F)$ & Details \\
\hline 4 & \\
3.5 & Higher than reference \\
2.84 & values \\
2.62 & \\
\hline 2.42 & \\
2.2 & Tolerance range \\
1.98 & \\
\hline 1.76 & \\
1.54 & Lower than reference \\
1.32 & values \\
1 & \\
0.1 & \\
\hline
\end{tabular}

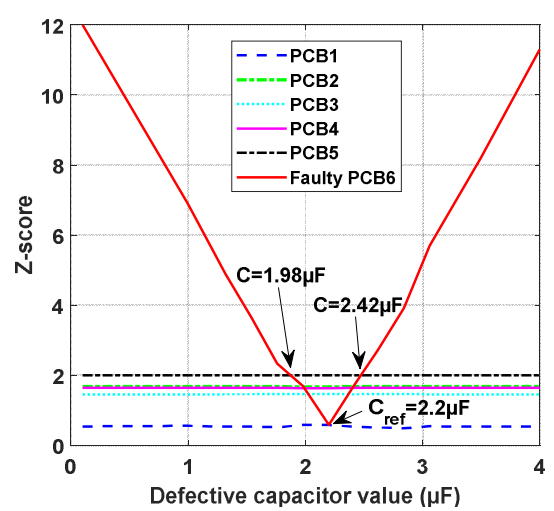

Fig. 16. Z-score evolution of all PCBs for different defect capacitor values

Figure 16 shows that the Z-score of the faulty PCB6 evolves nearly symmetrically about the reference value which means that whether the faulty value is higher or lower than reference the detection of the outlier PCB is assured. Furthermore and most importantly, this figure shows the threshold of outlier detection, which is a Z-score value that equals two. In other words, the calculated distance from the centroid of the non-faulty PCB cluster must be higher than twice the standard deviation of this cluster in order to insure a good detection of an outlier PCB. With this being the worst case defect scenario, the detection using this z-score criterion is always true for all other defect scenarios mentioned in Table 6.

\subsection{Defected components detection on a faulty} $P C B$

As mentioned earlier, PC scores are the new coordinates of the data matrix in the PC coordinates system defined by the 'Loadings', which is a square matrix containing the PC vectors. The transpose of this matrix gives the initial coordinate system vectors (IV) coordinates in the new PC coordinate system. 
These projected vectors give information about the directions in which data is scattered as well as the contribution of each principal component in the scattering. This will be shown through the defect cases presented next, as we project the transpose of the 'Loadings': Lo1, Lo2, Lo3, and Lo4 in the 3D score plot.

\section{- Defect scenario $N^{\circ}$ 1: One wrong value capacitor detection:}

A data matrix was generated containing a wrong value capacitor C8 inserted in PCB6. After running a PCA on the data, results for both cases $a$ and $b$ show that PCB6 is isolated from the non-faulty PCB cluster. As the calculated Z-score values in both cases are higher than the threshold, PCB6 is considered an outlier, thus, a defected PCB.

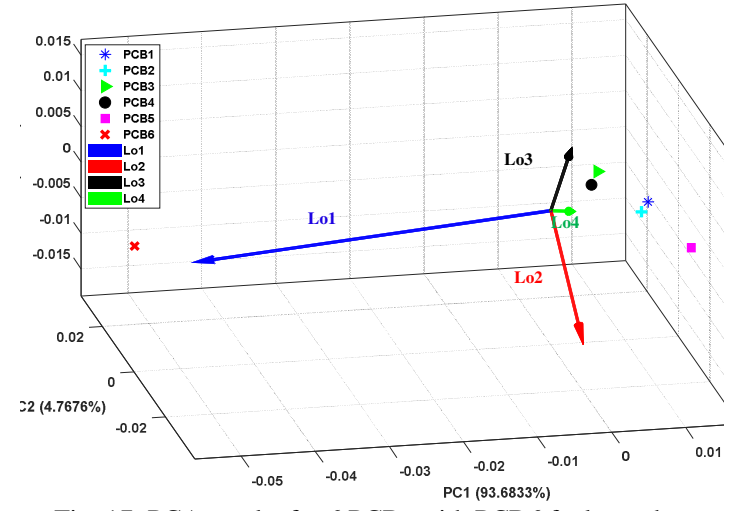

Fig. 17. PCA results for 6 PCBs with PCB6 faulty and one defected capacitor $\mathrm{C} 8=0.1 \mu \mathrm{F} / \mathrm{Z}$-score $=12.38$

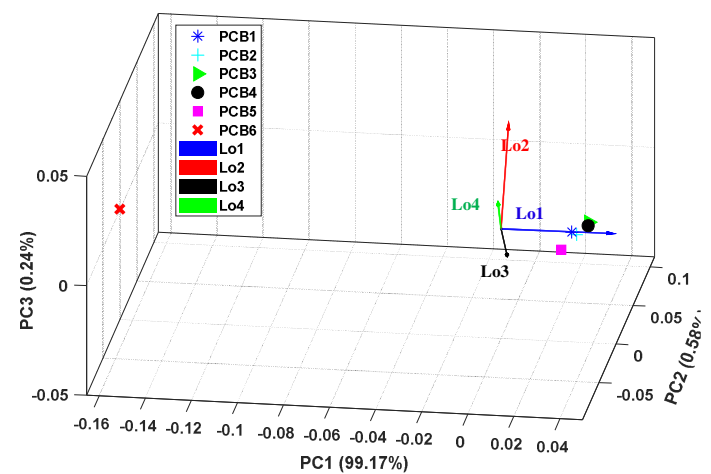

Fig. 18. PCA results for $6 \mathrm{PCBs}$ with PCB6 faulty and one defected capacitor $\mathrm{C} 8=8 \mu \mathrm{F} / \mathrm{Z}$-score $=12.39$

For the first case where $\mathrm{C} 8$ has a value lower than reference $\left(\mathrm{C}_{\mathrm{ref}}=2.2 \mu \mathrm{F}\right)$ : the projection of the IVs shows that only Lol, which is the projection corresponding to the first $\mathrm{PC}$ in the $\mathrm{PC}$ space, points toward the defect plane which is the plane containing the faulty PCB6 (see Fig.17). Contrarily to the second case where $\mathrm{C} 8$ has a value higher than $\mathrm{C}_{\text {ref }}$, Lol points away from the defect plane (see Fig.18).

\section{- Defect scenario $N^{\circ}$ 2: All wrong value capacitors}

\section{detection:}

Similarly to case $a$ and $b$, when all defected capacitors have lower values than the reference (case $c$ ) all the projected IVs point toward the defect plane (see Fig. 19). Figure 20 shows that values higher than the reference (case $d$ ) leads also to projected IVs pointing away from this plane.

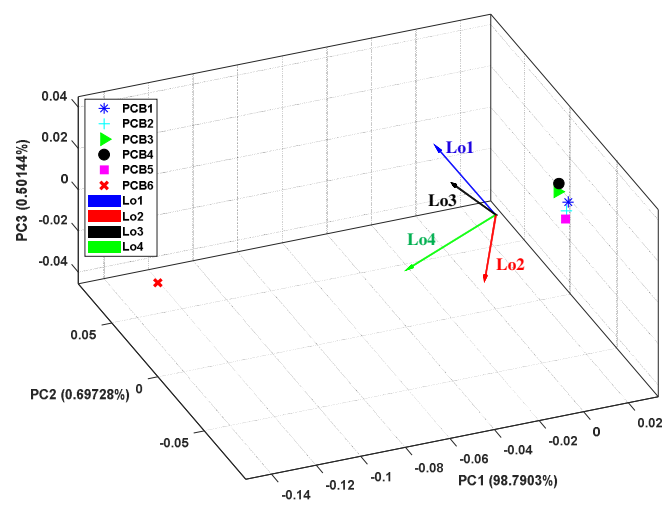

Fig. 19. PCA results for 6 PCBs with PCB6 faulty and all defected capacitors $\mathrm{C} 8=0.2 \mu \mathrm{F}, \mathrm{C} 9=0.5 \mu \mathrm{F}, \quad \mathrm{C} 10=0.3 \mu \mathrm{F}$ and $\mathrm{C} 11=0.1 \mu \mathrm{F} / \mathrm{Z}$-score $=33.22$

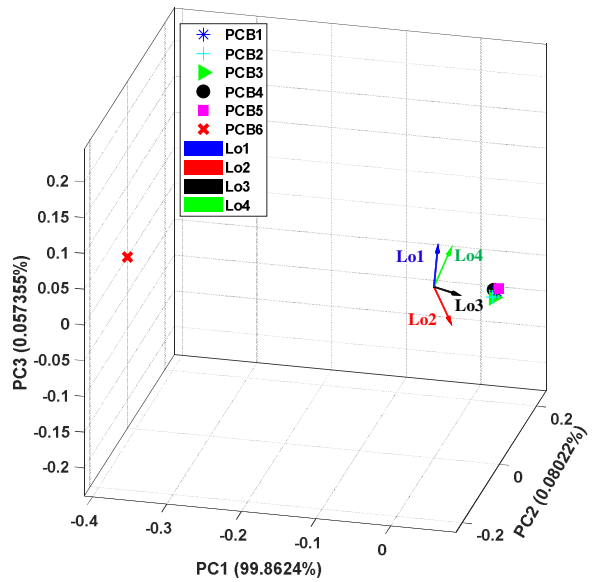

Fig. 20. PCA results for 6 PCBs with PCB6 faulty and all defected capacitors $\mathrm{C} 8=22 \mu \mathrm{F}, \mathrm{C} 9=33 \mu \mathrm{F}, \mathrm{C} 10=40 \mu \mathrm{F}$ and $\mathrm{C} 11=10 \mu \mathrm{F} / \mathrm{Z}$-score $=81.58$

In case $e$, only capacitor $\mathrm{C} 9$ has a wrong value lower than reference, the others have higher values. Lo2, which is the projected IV corresponding to the second PC, points toward the defect plane (see Fig. 21).

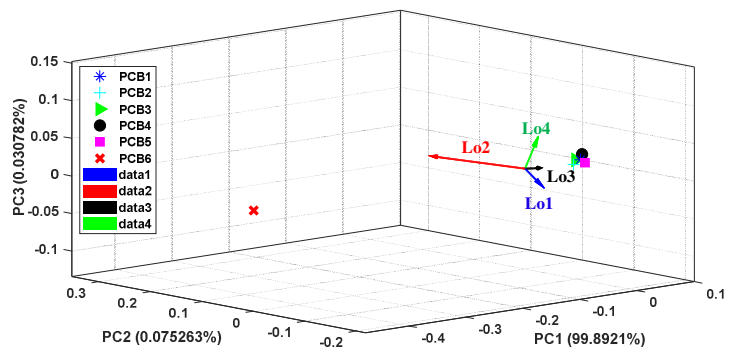

Fig. 21. PCA results for 6 PCBs with PCB6 faulty and all defected capacitors $\mathrm{C} 8=22 \mu \mathrm{F}, \mathrm{C} 9=1 \mu \mathrm{F}, \mathrm{C} 10=40 \mu \mathrm{F}$ and $\mathrm{C} 11=10 \mu \mathrm{F} / \mathrm{Z}$-score $=104.42$

Capacitors C8, C9 and C11, which have lower values than the reference, have their corresponding projected IVs Lo1, Lo2 and Lo4 pointing directly to the defect plane. Lo3 corresponding to C10, which has a higher value than the reference, points away from this plane (see Fig. 22). 


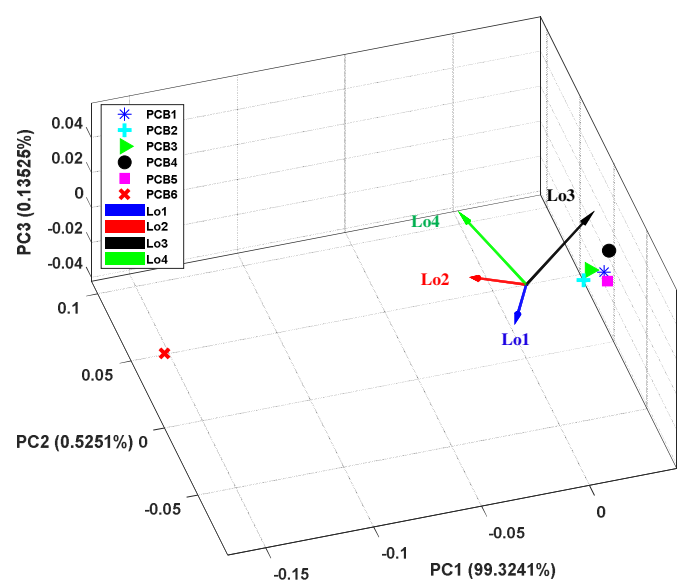

Fig. 22. PCA results for 6 PCBs with PCB6 faulty and all defected capacitors $\mathrm{C} 8=0.2 \mu \mathrm{F}, \mathrm{C} 9=0.5 \mu \mathrm{F}, \mathrm{C} 10=5 \mu \mathrm{F}$ and $\mathrm{C} 11=0.1 \mu \mathrm{F} / \mathrm{Z}$-score $=38.56$

Defected PCBs have always a Z-score higher than the threshold. They are a part of the defect plane formed by the axes PC2 and PC3 at the PC1 coordinate of the defected PCB. Projected IVs pointing toward this plane correspond to defected capacitors that have a lower value than reference. Those that point away from the defect plane correspond to either wrong value capacitors that have higher values than the reference (see Fig. 21), or nonfaulty in-tolerance capacitors (see Fig. 17).

The use of PCA and Z-score allows not only a detection of a faulty PCB, but also the components that might be contributing to this defect. Knowing that an operator must intervene to change the defected components indicated by projected PC vectors pointing toward the defect plane, a quick check on components corresponding to the other vectors is necessary.

\section{Discussion:}

The investigation of the approach presented in this paper aims to upgrade in-circuit testers by replacing some of the ICT classical nails used to perform electrical test of the components mounted on the board, with contactless probes integrating magnetic field (MF) sensors which measure the near MF over the components and give on-line assembly defect detection. The main advantages of this approach consists in:

- Granting physical accessibility to test mounted components using a minimum of test points

- PCB surface reduction

- Lowering the contact nails and test point count on the board

- Reducing the parasitic influence of the nail on the measured signal (i.e. less degradation of signal integrity of high speed digital signals)

Considerations that must be taken into account when using this technique are:

- Powering the tested block in order to get measurable magnetic signatures over the components

- Only components which have a significant
MF emission in the near field region are testable: we proved that this approach works for both ceramic and tantalum capacitors and inductors

- DFT rules establishment is a primary phase that must be set-up upstream with the design department in order to determine the powering conditions of the component blocks to be tested.

Economically speaking, the use of probes integrating magnetic sensors will be profitable compared to the use of the classical ICT nails in terms of the number of the probes used and the saved area on the PCB. Nevertheless, a detailed return on investment study should be conducted in order to quantify this profit.

\section{Conclusion}

We presented the possibility of using magnetic field signatures to diagnose faulty components contactlessly on a limited physical access PCBA. This approach proposed to overcome the limitations of classical ICT, uses a detection algorithm based on Principal Component Analysis and Z-score to reinforce the fault detection on a powered block of the BUT.

In the first part of the paper, we validated the principle of our proposed measurement approach through the test of the filtering components of a DC/DC converter. Miniature near field probes and GMR sensors were used to measure magnetic field distributions over powered sensitive components and to give insight on the value of the component and its solder condition (soldered or non-soldered). The loading of the BUT was specifically chosen to enhance the sensitivity of the EM measurements.

These first experimental results demonstrate that the magnetic field probing approach can provide a viable option to detect specific component level defects and decrease the number of traditional test points while still providing access. However, the magnetic signature measurement must be coupled to a robust defect detection method.

The outlier detection approach used in this first part was a simple statistical indicator that compares the deviations of measurements around reference signatures. It turned to be not really significant in complex defect scenarios which may exist in a typical industrial PCBA.

That's why we introduced the use of Principal Component Analysis algorithm in the second part of the article. Its purpose is not only the detection of defected PCBAs but also the identification of the components responsible for the defect. Using data measurements from Monte-Carlo simulations run on our DC/DC buck converter model, demonstrated that this algorithm can give excellent results regarding the detection of the defected PCBAs and the components responsible for their defect.

Limitation of this algorithm in faulty component identification may appear in some particular combination of defect components. For example, simulation results showed that, when the defected capacitors had both higher and lower values 
than the reference ones, the identification of faulty or non-faulty components can be wrong. However, the algorithm identifies the faulty nature of the PCB successfully.

Research is still on-going to improve the measurement technique and the detection method with the aim of a full integration in industrial ICT. The perspectives of this work include:

- The application of this test approach to other types of circuits in order to determine all the possible cases of application.

- Improving the measurement sensitivity and detection reliability.

- Associating the detection method with a machine learning algorithm allowing the in-circuit tester to better detect defects throughout the test of a series of the same product.

\section{Acknowledgments}

This work was supported by the National Association of Research and Technology (ANRT) and the characterization platform of LAAS-CNRS.

\section{References}

[1] Robinson,Verma, "Optimizing Test Strategies during PCB Design For Boards with Limited ICT Access", Proceedings of the Telecom Hardware Solutions Conference, May 2002.

[2] D.Gizopoulos, Advances in ElectronicTesting: Challenges and Methodologies. New York: Springer, 2006. P.371.

[3] N. El Belghiti Alaoui, P. Tounsi, A. Boyer and A. Viard, "New testing approach using near electromagnetic field probing intending to upgrade in-circuit testing of high density PCBAs," 2018 IEEE 27th North Atlantic Test Workshop (NATW), Essex, VT, 2018, pp. 1-8

[4] Texas Instruments web site, Power management:http://www.ti.com/lit/ug/snva285a /snva285a.pdf 2013 (accessed 10 July 2018)

[5] Allegro Design Entry CIS (Cadence Design systems, 17.2-2016)

[6] H-Field Probe mini $30 \mathrm{MHz}$ up to $3 \mathrm{GHz}$, Langer EMV Technik, more information on www.langer-emv.de

[7] A. Bernieri, G. Betta, L. Ferrigno, M. Laracca, "Improving performance of GMR sensors", IEEE Sensors J., vol. 13, no. 11, pp. 45134521, Nov. 2013.

[8] NVE web site, Analog products: https://www.nve.com/Downloads/analog_catao g.pdf 2018 (accessed 10 July 2018)

[9] Texas Instruments web site, Power management: http://www.ti.com/lit/ug/slvuao9b/slvuao9b.pdf 2017 (accessed 10 July 2018)

[10] Texas instruments web site, power management products:http://www.ti.com/lit/an/slta055/slta05 5.pdf 2006 (accessed 26 June 2018)

[11] V.Barnett, T.Lewis, 1994, Outliers in statistical data, Wiley; 3rd edition

[12] J. Rousseeuw, M. Leroy, 2003, Robust regression and Outlier Detection, WileyInterscience; 1 st edition
[13] A.Campbell, N \& Atchley, William. (1981). The Geometry of Canonical Variate Analysis. Systematic Zoology. vol.30, p.268-272

[14] Statistics and Machine Learning Toolbox (Matlab, R2017b (9.3.0.713579))

[15] Lin L, Sherman PD. Cleaning Data the Chauvenet Way. SouthEast SAS Users Group. Hilton Head, SC: Institute for Advanced Analytics; 2007 